PROCEEDINGS OF THE

AMERICAN MATHEMATICAL SOCIETY

Volume 128 , Number 12, Pages $3731-3740$

S 0002-9939(00)05444-7

Article electronically published on June 14, 2000

\title{
SOME NEW EMBEDDINGS AND NONIMMERSIONS OF REAL PROJECTIVE SPACES
}

\author{
DONALD M. DAVIS AND VITALY ZELOV
}

(Communicated by Ralph Cohen)

\begin{abstract}
We obtain new families of embeddings and nonimmersions of real projective spaces in Euclidean space. The method involves obstruction theory, and includes several new insights.
\end{abstract}

\section{Statement of Results}

In this paper, we obtain the following new results regarding immersions and embeddings of real projective space $P^{m}$ in Euclidean space. Let $\alpha(n)$ denote the number of 1's in the binary expansion of $n$.

Theorem 1.1. If $\alpha(n)=2$, then

(1) $P^{16 n+8}$ cannot be immersed in $\mathbf{R}^{32 n+3}$, and

(2) $P^{16 n+10}$ cannot be immersed in $\mathbf{R}^{32 n+11}$.

Theorem 1.2. If $\alpha(n)>2$, then $P^{8 n+4}$ can be embedded in $\mathbf{R}^{16 n+1}$.

Theorem 1.11) improves on the previously best known result (1]) by 1 dimension, while Theorem 1.1(2) improves on the previously best known nonimmersion and nonembedding results (2]) for $P^{16 n+10}$ and $P^{16 n+11}$ by 4 dimensions, and is within 1 of best possible for them. It also implies new nonimmersions for $P^{16 n+12}$, $P^{16 n+13}$, and $P^{16 n+14}$. Theorem 1.2 improves on the previously best known embedding ([11]) of $P^{8 n+4}$ by 1 dimension. These results can best be appreciated when viewed in a table of known embedding and immersion results for real projective spaces. Such a table may be seen on the internet (see [3]).

The method of proof is obstruction theory, specifically modified Postnikov towers (MPTs). The reason that Theorem 1.1 had not been noticed before is that the first author used to think that nonimmersions were extremely difficult to prove by MPTs because of the possibility of secondary and higher order indeterminacy. We show here that sometimes there is no secondary or higher order indeterminacy for a simple reason. The reason that Theorem 1.2 had not been noticed before is that this was apparently the first time that Mahowald's inductive approach to constructing embeddings ([8]) was combined with the method of evaluation of obstructions initiated in [5].

Received by the editors February 12, 1999.

1991 Mathematics Subject Classification. Primary 57R40.

Key words and phrases. Embeddings, immersions, real projective spaces, obstruction theory.

(C)2000 American Mathematical Society 
Theorem 1.1(1) and Theorem 1.2 form the bulk of the second author's thesis ([12]), written under the direction of the first author, who subsequently discovered the second part of Theorem 1.1

\section{Proof of NONIMMERsions}

In this section, we prove Theorem 1.1(2). The proof of Theorem 1.1(1), which was detailed in $[12$, is extremely similar, and is omitted.

The problem is reduced to obstruction theory by the following result of Sanderson, which reduces the immersion question to the determination of the geometric dimension of a multiple of the Hopf bundle $\xi_{n}$ over $P^{n}$.

Proposition 2.1 ([10]). $P^{n}$ can be immersed in $\mathbf{R}^{n+k}$ if and only if the map $P^{n} \rightarrow$ $B O$ which classifies $(n+k+1) \xi_{n}$ can be factored as $P^{n} \rightarrow B O(k) \rightarrow B O$.

Thus Theorem 1.1(2) will follow from the following result, whose proof will occupy the rest of this section.

Theorem 2.2. If $\alpha(n)=2$, then $(32 n+12) \xi: P^{16 n+10} \rightarrow B O$ does not factor through $B O(16 n+1)$.

The map of Theorem 2.2 factors as

$$
P^{16 n+10} \rightarrow H P^{4 n+2} \stackrel{(8 n+3) H}{\rightarrow} B S p \rightarrow B O .
$$

Here $H P^{m}$ denotes quaternionic projective space, and $p H$ a multiple of the quaternionic Hopf bundle. We let $\widetilde{B S p}(n)$ denote the classifying space for quaternionic bundles of real geometric dimension $n$; it is the pullback of maps of $B O(n)$ and $B S p$ to $B O$. We will prove Theorem 2.2 by showing that the map $P^{16 n+10} \rightarrow B S p$ in (2.3) does not lift to $\widetilde{B S p}(16 n+1)$. This will be accomplished using the following MPT. For typographical reasons, we abbreviate $K\left(\mathbf{Z}_{2}, 16 n+i\right)$ as $K_{i}$. We will, however, name the corresponding $k$-invariant $k_{16 n+i}^{j} \in H^{16 n+i}\left(E_{j}\right)$. (All coefficients are in $\mathbf{Z} / 2$.) All of our MPTs are performed through the range of dimensions relevant for the real projective space being mapped in, here $16 n+10$. This will always be well within the stable range.

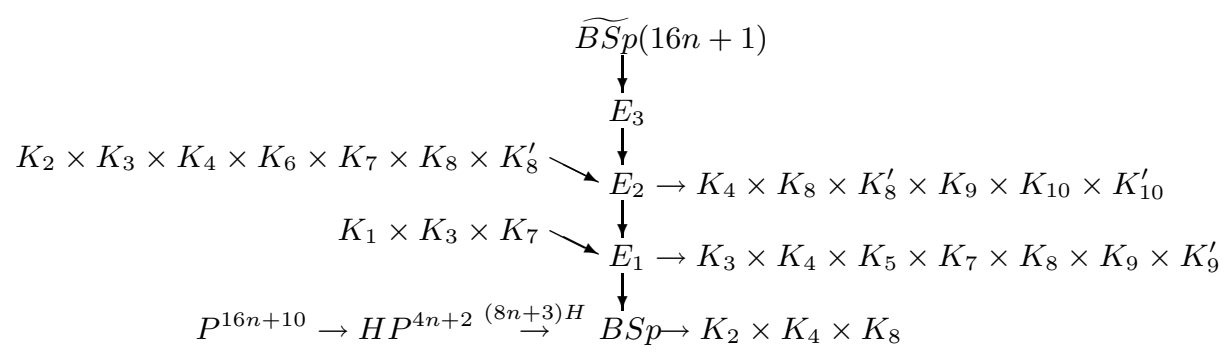

\section{FiguRe 2.4 .}

MPTs were introduced in [8] and [6]. Each vertical map in the above diagram is part of a fiber sequence preceded by the map from the fiber represented by a diagonal arrow, and followed by the classifying map represented by a horizontal arrow. The information of the diagram can be obtained from the ASS of the stunted real projective space $P_{16 n+1}$, which is, in the stable range, the fiber of 
$\widetilde{B S p}(16 n+1) \rightarrow B S p$. This ASS can be found in Table 8.2 on page 54 of $[9]$. We re-create it in Figure [2.5.

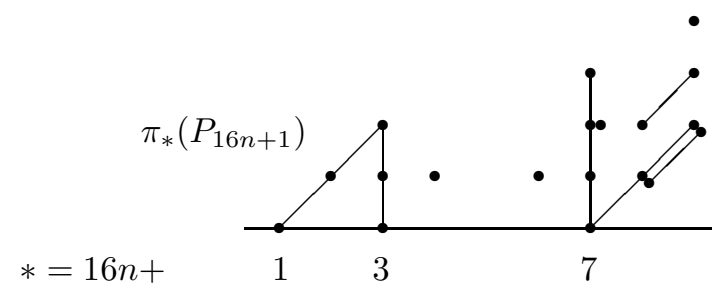

FIGURE 2.5 .

The generalized Eilenberg-MacLane spaces (GEMs) on the right side of Figure 2.4 give rise to the $k$-invariants for the MPT; these classes have dimensions 1 greater than those of the corresponding elements of $\pi_{*}\left(P_{16 n+1}\right)$. We will prove that the map $P^{16 n+10} \rightarrow B S p$ lifts to $E_{2}$, and every lifting to $E_{2}$ sends some of the level-2 $k$-invariants nontrivially. Hence the map does not lift to $E_{3}$ or to $\widetilde{B S p}(16 n+1)$.

The method of evaluation of obstructions is to use the following result of [5]. Here $b o$ is the spectrum for connective $k o$-theory localized at 2.

Theorem 2.6 ([5, 1.8]). Let $B^{o}(m)$ denote the fiberwise smash product of $\widetilde{B S p}(m)$ with bo. In the stable range, there is a map of fibrations which is the natural inclusion on the fibers

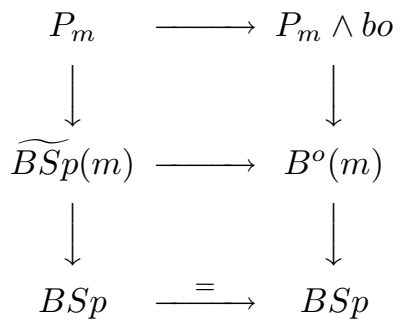

If $p H_{k}$ denotes $p$ times the Hopf bundle over $H P^{k}$, then $p H_{k}$ lifts to $B^{o}(m)$ if and only if $m \geq 2 k$ and for all $i \leq k$

$$
\nu\left(\left(\begin{array}{l}
p \\
i
\end{array}\right)\right) \geq \nu\left(\left|k o_{4 i-1}\left(P_{m}\right)\right|\right) .
$$

Here, and throughout, $\nu(-)$ denotes the exponent of 2 .

We use Theorem 2.6 to prove the following result.

Theorem 2.7. In the MPT of Figure 2.4, there is a lifting of $(8 n+3) H_{4 n+2}$ to $E_{2}$ sending $k_{16 n+4}^{2}$ and $k_{16 n+8}^{2}$ nontrivially.

Proof. We begin by observing that $\nu\left(\begin{array}{l}8 n+3 \\ 4 n+1\end{array}\right)=\nu\left(\begin{array}{l}8 n+3 \\ 4 n+2\end{array}\right)=\alpha(n)=2$. Indeed, both are equal to $\alpha(4 n+1)+\alpha(4 n+2)-\alpha(8 n+3)$.

These must be compared with the numbers in the following table. Results such as these have been tabulated in many papers of the first author, such as [5]. 


\begin{tabular}{r|cccc|} 
& \multicolumn{4}{|c|}{$m$} \\
$\nu\left(k o_{4 i-1}\left(P_{m}\right)\right)$ & $16 n+1$ & $16 n+2$ & $16 n+5$ & $16 n+6$ \\
\hline$i=4 n+1$ & 3 & 2 & 0 & 0 \\
$i=4 n+2$ & 4 & 4 & 3 & 2 \\
\hline
\end{tabular}

The orders tabulated here correspond to ASS charts pictured below, which may be computed, for example, as in [4].

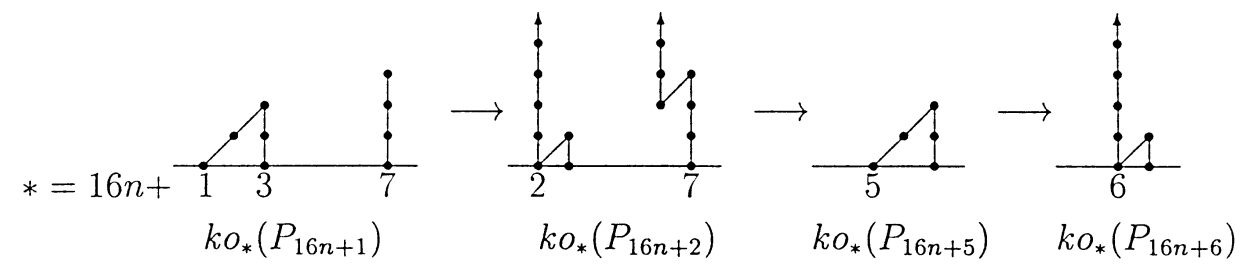

FIGURE 2.8 .

There are MPTs for the fibrations $B^{\circ}(16 n+\delta) \rightarrow B S p$, and induced maps of the MPTs, whose spaces we denote by $E_{s}^{o}(16 n+\delta)$. By Theorem 2.6 $(8 n+3) H_{4 n+1}$ lifts to $B^{o}(16 n+2)$ but not to $B^{o}(16 n+1)$. This implies that $(8 n+3) H_{4 n+1}$ lifts to $E_{2}^{o}(16 n+1)$ sending $k_{16 n+4}^{2}$ nontrivially. (Keep in mind that degrees of $k$-invariants are 1 greater than those of corresponding elements of $\pi_{*}$ (fiber), which in this case is $k o_{*}\left(P_{16 n+\delta}\right)$.) Similarly, $(8 n+3) H_{4 n+2}$ lifts to $B^{o}(16 n+6)$, but not to $B^{o}(16 n+5)$. All this implies that $(8 n+3) H_{4 n+2}$ lifts to $E_{2}^{o}(16 n+1)$, sending both $k_{16 n+4}^{2}$ and $k_{16 n+8}^{2}$ nontrivially.

The map $\widetilde{B S p}(16 n+1) \rightarrow B^{o}(16 n+1)$ induces a map of MPTs. The mapping of $k$-invariants corresponds to the map of fibers $\pi_{*}\left(P_{16 n+1}\right) \rightarrow k o_{*}\left(P_{16 n+1}\right)$, which are pictured in Figures 2.5] and2.8. Various methods of computing Ext homomorphisms can be used to show this morphism is surjective in the indicated range. If $F$ denotes the fiber of $E_{2} \rightarrow E_{2}^{o}(16 n+1)$, then $\pi_{*}(F)$ corresponds to elements in filtration 0 and 1 in Figure 2.5 which map trivially to $k o_{*}\left(P_{16 n+1}\right)$. Such elements occur only in filtration 1 and in homotopy dimension $16 n+4,16 n+6$, and $16 n+8$. The obstructions to pulling the map $H P^{4 n+2} \rightarrow E_{2}^{o}(16 n+1)$ back to $E_{2}$ occur in $H^{*}\left(H P^{4 n+2} ; \pi_{*-1}(F)\right)$, which is 0 since $\pi_{*-1}(F)=0$ when $* \equiv 0 \bmod 4$ and $* \leq 16 n+8$. Thus $(8 n+3) H_{4 n+2}$ lifts to $E_{2}$. The $k$-invariants $k_{16 n+4}^{2}$ and $k_{16 n+8}^{2}$ in $H^{*}\left(E_{2}\right)$ are the images of corresponding $k$-invariants in $H^{*}\left(E_{2}^{o}(16 n+1)\right)$, which have already been shown to map nontrivially to $H^{*}\left(H P^{4 n+2}\right)$. Thus $k_{16 n+4}^{2}$ and $k_{16 n+8}^{2}$ map nontrivially, as claimed. We make no claim about whether or not $k_{16 n+8}^{2^{\prime}}$, which corresponds to the split $\mathbf{Z} / 2$ in $\pi_{16 n+7}\left(P_{16 n+1}\right)$ in filtration 2 , maps nontrivially, since it is not in the image from $H^{*}\left(E_{2}^{o}(16 n+1)\right)$.

In order to determine the indeterminacy for lifting $P^{16 n+10}$ in this MPT, we must know the relations which give rise to the $k$-invariants. These are computed by the method initiated in [6] and utilized in many subsequent papers by the first author and also in papers of Lam and/or Randall. It is a matter of building a minimal resolution using Massey-Peterson algebras. The relations for the MPT in Figure 2.4 are given in the table below. 


\begin{tabular}{|ll|}
\hline$w_{16 n+2}$ & \\
$w_{16 n+4}$ & \\
$w_{16 n+8}$ & \\
\hline$k_{16 n+3}^{1}:$ & $\mathrm{Sq}^{2} w_{16 n+2}$ \\
$k_{16 n+4}^{1}:$ & $\mathrm{Sq}^{1} w_{16 n+4}+\mathrm{Sq}^{2} \mathrm{Sq}^{1} w_{16 n+2}$ \\
$k_{16 n+5}^{1}:$ & $\left(\mathrm{Sq}^{4}+w_{4}\right) w_{16 n+2}$ \\
$k_{16 n+7}^{1}:$ & $\left(\mathrm{Sq}^{4}+w_{4}\right) w_{16 n+4}$ \\
$k_{16 n+8}^{1}:$ & $\mathrm{Sq}^{1} w_{16 n+8}+\mathrm{Sq}^{2} \mathrm{Sq}^{3} w_{16 n+4}$ \\
$k_{16 n+9}^{1}:$ & $\mathrm{Sq}^{2} w_{16 n+8}+\left(\mathrm{Sq}^{4}+w_{4}\right) \mathrm{Sq}^{2} w_{16 n+4}$ \\
$k_{16 n+9}^{\prime}:$ & $\left(\mathrm{Sq}^{8}+w_{8}\right) w_{16 n+2}+w_{4} \mathrm{Sq}^{2} w_{16 n+4}$ \\
\hline$k_{16 n+4}^{2}:$ & $\mathrm{Sq}^{2} k_{16 n+3}^{1}+\mathrm{Sq}^{1} k_{16 n+4}^{1}$ \\
$k_{16 n+8}^{2}:$ & $\mathrm{Sq}^{1} k_{16 n+8}^{1}+\mathrm{Sq}^{2} \mathrm{Sq}^{3} k_{16 n+4}^{1}$ \\
$k_{16 n+8}^{2^{\prime}}:$ & $\left(\mathrm{Sq}^{4}+\mathrm{Sq}^{3} \mathrm{Sq}^{1}+w_{4}\right) k_{16 n+5}^{1}+\left(\mathrm{Sq}^{6}+w_{4} \mathrm{Sq}^{2}\right) k_{16 n+3}^{1}$ \\
$k_{16 n+9}^{2}:$ & $\mathrm{Sq}^{2} \mathrm{Sq}^{1} k_{16+7}^{1}+\left(\mathrm{Sq}^{4}+w_{4}\right) \mathrm{Sq}^{1} k_{16 n+5}^{1}+\left(\mathrm{Sq}^{6}+w_{4} \mathrm{Sq}^{2}\right) k_{16 n+4}^{1}$ \\
& $+\left(\mathrm{Sq}^{4} w_{4}\right) \mathrm{Sq}^{2} \mathrm{Sq}^{1} k_{16 n+3}^{1}$ \\
$k_{16 n+10}^{2}:$ & $\mathrm{Sq}^{2} k_{16 n+9}^{1}+\mathrm{Sq}^{3} k_{16 n+8}^{1}+\left(\mathrm{Sq}^{4}+\mathrm{Sq}^{3} \mathrm{Sq}^{1}+w_{4}\right) k_{16 n+7}^{1}$ \\
$k_{16 n+10}^{2^{\prime}}:$ & $\mathrm{Sq}^{2} k_{16 n+9}^{1^{\prime}}+\left(\mathrm{Sq}^{5} \mathrm{Sq}^{1}+\mathrm{Sq}^{4} \mathrm{Sq}^{2}\right) k_{16 n+5}^{1}+w_{4} \mathrm{Sq}^{3} k_{16 n+4}^{1}$ \\
& $+\left(\mathrm{Sq}^{8}+w_{8}+w_{4} \mathrm{Sq}^{4}+w_{4}^{2}+w_{4} \mathrm{Sq}^{3} \mathrm{Sq}^{1}\right) k_{16 n+3}^{1}$ \\
\hline$k_{16 n+8}^{3}:$ & $\mathrm{Sq}^{1} k_{16 n+8}^{2}+\mathrm{Sq}^{2} \mathrm{Sq}^{3} k_{16 n+4}^{2}$ \\
$k_{16 n+10}^{3}:$ & $\mathrm{Sq}^{2} k_{16 n+9}^{2}+\mathrm{Sq}^{2} \mathrm{Sq}^{1} k_{16 n+8}^{2^{\prime}}$ \\
& $+\left(\mathrm{Sq}^{7}+\mathrm{Sq}^{4} \mathrm{Sq}^{2} \mathrm{Sq}^{1}+w_{4}\left(\mathrm{Sq}^{3}+\mathrm{Sq}^{2} \mathrm{Sq}^{1}\right)\right) k_{16 n+4}^{2}$ \\
\hline$k_{16 n+10}^{4}:$ & $\mathrm{Sq}^{2} \mathrm{Sq}^{1} k_{16 n+8}^{3}$ \\
\hline
\end{tabular}

The lifting $f_{2}: P^{16 n+10} \rightarrow E_{2}$ can be varied through the fiber $F_{1}$ of $E_{2} \rightarrow E_{1}$, which is the GEM in Figure 2.4 which ends with $K_{8}^{\prime}$. This primary indeterminacy is computed using the above relations. For the bundle $(32 n+12) \xi$, both $w_{4}$ and $w_{8}$ are nonzero. Varying through $K_{16 n+2}$ changes $f_{2}^{*}\left(k_{16 n+4}^{2}\right)$ and $f_{2}^{*}\left(k_{16 n+8}^{2^{\prime}}\right)$ and no other level-2 $k$-invariants. We illustrate why this is true in the second case.

The relation for $k_{16 n+8}^{2^{\prime}}$ means that the action map $\mu: F_{1} \times E_{2} \rightarrow E_{2}$ sends $k_{16 n+8}^{2^{\prime}}$ to

$$
\begin{aligned}
& 1 \otimes k_{16 n+8}^{2^{\prime}}+\left(\mathrm{Sq}^{4}+\mathrm{Sq}^{3} \mathrm{Sq}^{1}\right) \iota_{16 n+4} \otimes 1+\iota_{16 n+4} \otimes w_{4}+\mathrm{Sq}^{6} \iota_{16 n+2} \\
& \otimes 1+\mathrm{Sq}^{2} \iota_{16 n+2} \otimes w_{4} .
\end{aligned}
$$

The composite

$$
P^{16 n+10 \stackrel{x^{16 n+2}}{\rightarrow} \times f_{2}} F_{1} \times E_{2} \stackrel{\mu}{\rightarrow} E_{2}
$$

sends $k_{16 n+8}^{2^{\prime}}$ to

$$
f_{2}^{*}\left(k_{16 n+8}^{2^{\prime}}\right)+\mathrm{Sq}^{6}\left(x^{16 n+2}\right)+\mathrm{Sq}^{2}\left(x^{16 n+2}\right) \cdot x^{4}=f_{2}^{*}\left(k_{16 n+8}^{2^{\prime}}\right)+0+x^{16 n+8} .
$$

Thus (2.9) would be a new lifting to $E_{2}$ with $f_{2}^{*}\left(k_{16 n+8}^{2^{\prime}}\right)$ changed. A similar computation is required to determine whether (2.9) changes each of the other $k$-invariants, and the result is as claimed in the previous paragraph.

Similarly, varying through $K_{16 n+3}$ changes $f_{2}^{*}\left(k_{16 n+4}^{2}\right), f_{2}^{*}\left(k_{16 n+8}^{2}\right), f_{2}^{*}\left(k_{16 n+9}^{2}\right)$, and $f_{2}^{*}\left(k_{16 n+10}^{2^{\prime}}\right)$. These occur because the following terms, respectively, are nonzero: 
$\mathrm{Sq}^{1} x^{16 n+3}, \mathrm{Sq}^{2} \mathrm{Sq}^{3}\left(x^{16 n+3}\right), w_{4} \mathrm{Sq}^{2}\left(x^{16 n+3}\right)$, and $w_{4} \mathrm{Sq}^{3}\left(x^{16 n+3}\right)$. The only other way in which $f_{2}^{*}\left(k_{16 n+\delta}^{2}\right)$ can be changed is by varying through $K_{16 n+7}$, which changes $f_{2}^{*}\left(k_{16 n+8}^{2}\right)$ and $f_{2}^{*}\left(k_{16 n+10}^{2}\right)$.

Thus varying the lifting through $F_{1}$ in such a way that both $f_{2}^{*}\left(k_{16 n+4}^{2}\right)$ and $f_{2}^{*}\left(k_{16 n+8}^{2}\right)$ are changed to become 0 will cause either $f_{2}^{*}\left(k_{16 n+10}^{2}\right)$ or $f_{2}^{*}\left(k_{16 n+10}^{2^{\prime}}\right)$ to become nonzero. Thus if $f_{1}: P^{16 n+10} \rightarrow E_{1}$ represents a lifting obtained by factoring through $H P^{4 n+2}$, then every lifting of $f_{1}$ to $E_{2}$ sends some $k$-invariants nontrivially, and hence $f_{1}$ does not lift to $\widetilde{B S p}(16 n+1)$.

We must also consider the possibility that $f_{1}$ could be varied through the fiber, $F_{0}$, of the map $E_{1} \rightarrow B S p$ in such a way that the new map $f_{1}^{\prime}$ lifted to $f_{2}^{\prime}$ : $P^{16 n+10} \rightarrow E_{2}$ and sent all $k^{2}$-invariants to 0 . This is the secondary indeterminacy consideration that had led the first author to not try to prove nonimmersions by ordinary MPTs in his work during the 1970s and 1980s. However, in the case at hand, secondary indeterminacy is not a problem because any nontrivial map $P^{16 n+10} \rightarrow F_{0}$ will change the images of some of the $k^{1}$-invariants. Since $f_{1}$ was a map which lifted to $E_{2}$ and therefore sent all the $k^{1}$-invariants to 0 , any variation of $f_{1}$ through $F_{0}$ will not lift to $E_{2}$.

This completes the proof that $(32 n+12) \xi_{16 n+10}$ does not lift to $\widetilde{B S p}(16 n+1)$ when $\alpha(n)=2$, once we verify the statement made in the preceding paragraph about varying maps through $F_{0}$. Varying through $K_{16 n+1}$ changes $f_{1}^{*}\left(k_{16 n+4}^{1}\right)$, $f_{1}^{*}\left(k_{16 n+5}^{1}\right)$, and $f_{1}^{*}\left(k_{16 n+9}^{1^{\prime}}\right)$. Varying through $K_{16 n+3}$ changes $f_{1}^{*}\left(k_{16 n+4}^{1}\right)$, $f_{1}^{*}\left(k_{16 n+7}^{1}\right), \quad f_{1}^{*}\left(k_{16 n+8}^{1}\right)$, and $f_{1}^{*}\left(k_{16 n+9}^{1^{\prime}}\right)$. Varying through $K_{16 n+7}$ changes $f_{1}^{*}\left(k_{16 n+8}^{1}\right)$ and $f_{1}^{*}\left(k_{16 n+9}^{1}\right)$. Any nontrivial combination of these changes some $f_{1}^{*}\left(k_{16 n+\delta}^{1}\right)$, as claimed.

\section{Proof of EMbeddings}

In this section, we prove Theorem 1.2 We use the following result of Mahowald, which deals with topological embeddings.

Theorem 3.1 ([8]). Assume that $P^{q}$ embeds in $\mathbf{R}^{p}$ with normal bundle $\nu$.

- If $\nu \otimes \xi_{q}$ has $n$ linearly independent sections and $P^{n-1}$ embeds in $S^{m-1}$, then $P^{n+q}$ embeds in $\mathbf{R}^{p+m}$.

- $\nu \otimes \xi_{q} \oplus(q+1) \epsilon \approx(p+1) \xi_{q}$.

We apply this result to the embedding of $P^{8 n+2}$ in $\mathbf{R}^{16 n-1}$ when $\alpha(n)>2$, as proved in [11]. Using also the embedding of $P^{1}$ in $S^{1}$, we obtain Theorem 1.2 once we prove the following result. Here $\theta=\nu \otimes \xi_{8 n+2}$.

Theorem 3.2. If $\theta$ is an $(8 n-3)$-plane bundle over $P^{8 n+2}$ which is stably equivalent to $16 n \xi_{8 n+2}$, and $\alpha(n)>2$, then $\theta$ has at least 2 linearly independent sections.

Theorem 3.1 will then imply that there is a topological embedding of $P^{8 n+4}$ in $\mathbf{R}^{16 n+1}$. Such an embedding can be approximated by a differentiable embedding by a result of Haefliger $([7])$, since $2(16 n+1) \geq 3(8 n+4)$.

The first step toward proving Theorem 3.2 is to prove the following lemma.

Lemma 3.3. If $\alpha(n)>2$, the map $P^{8 n+2} \rightarrow B S p$ which classifies $16 n \xi_{8 n+2}$ lifts to $\widetilde{B S p}(8 n-5)$. 
Proof. Similar to the work of the previous section, we consider the following diagram.

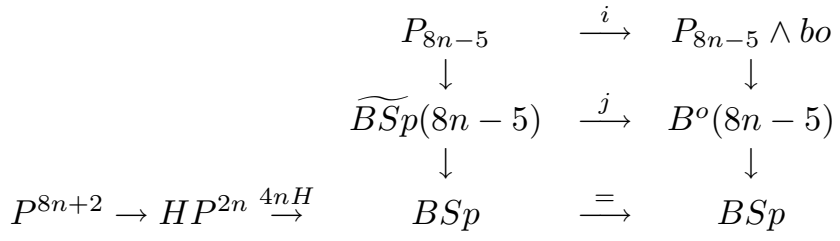

The morphism $\pi_{*}\left(P_{8 n-5}\right) \rightarrow k o_{*}\left(P_{8 n-5}\right)$ of homotopy groups of fibers is depicted in the following ASS charts. Again, the first is from 9] while the second is well known, e.g. 4]. The morphism is easily seen to be surjective.

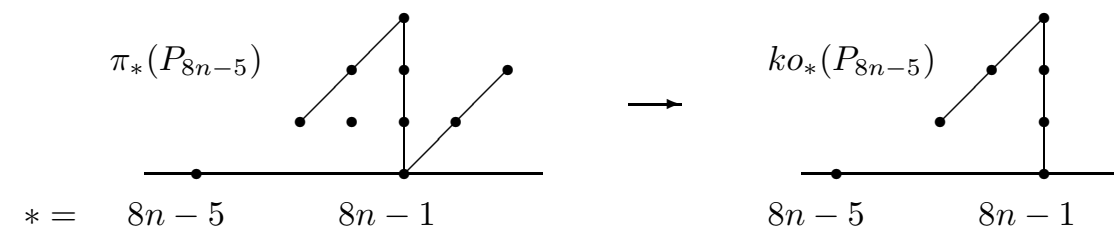

From the chart, we see that $\nu\left(\left|k o_{8 n-5}\left(P_{8 n-5}\right)\right|\right)=1$, and $\nu\left(\left|k o_{8 n-1}\left(P_{8 n-5}\right)\right|\right)=4$. One easily calculates $\nu\left(\left(\begin{array}{c}4 n \\ 2 n-1\end{array}\right)\right)>2$ and $\nu\left(\left(\begin{array}{c}4 n \\ 2 n\end{array}\right)\right)=\alpha(n)$. If $\alpha(n)>3$, then, by Theorem 2.6, $4 n H_{2 n}$ lifts to a map $\ell: H P^{2 n} \rightarrow B^{o}(8 n-5)$. Let $F=\operatorname{fiber}(j)=$ fiber $(i)$. Since $\pi_{*}(F)=0$ when $* \equiv 3 \bmod 4$ and $* \leq 8 n+2, \ell$ pulls back to the desired map $H P^{2 n} \rightarrow \widetilde{B S p}(8 n-5)$.

When $\alpha(n)=3$, we can show, similar to the proof of Theorem 2.7 that $4 n H_{2 n}$ lifts to $E_{3}^{o}(8 n-5)$, the third stage of the MPT for $B^{o}(8 n-5)$. This uses Theorem 2.6 to see that $4 n H_{2 n}$ lifts to $B^{\circ}(8 n-3)$ and $4 n H_{2 n-1}$ lifts to $B^{o}(8 n-5)$, which imply that the obstructions to lifting to $E_{3}^{o}(8 n-5)$ map trivially. The map $H P^{2 n} \rightarrow$ $E_{3}^{o}(8 n-5)$ pulls back to $E_{3}$, the third stage in the MPT for $\widetilde{B S p}(8 n-5)$, since $H^{*}\left(H P^{2 n} ; \pi_{*-1}(\right.$ fiber $\left.)\right)=0$.

The fiber of $E_{3} \rightarrow E_{2}$ contains a $K_{8 n-1}$-factor, corresponding to the third dot up in the ASS chart for $\pi_{8 n-1}\left(P_{8 n-5}\right)$. The map $f_{3}: R P^{8 n+2} \rightarrow H P^{2 n} \rightarrow E_{3}$ can be varied through this factor to change $f_{3}^{*}\left(k_{8 n}^{3}\right)$. This is implied by a $\mathrm{Sq}^{1} k_{8 n}^{2}$ which appears in the relation for $k_{8 n}^{3}$ in the appropriate MPT. Since $k_{8 n}^{3}$ is the only level-3 $k$-invariant, we deduce that there is a choice of the lifting $f_{3}$ which lifts to $E_{4}=\widetilde{B S p}(8 n-5)$.

The bundle $\theta$ with which we ultimately must deal might not be a symplectic bundle; however, it certainly is a Spin bundle. We reinterpret Lemma 3.3 to say

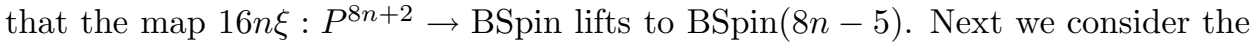
following diagram.

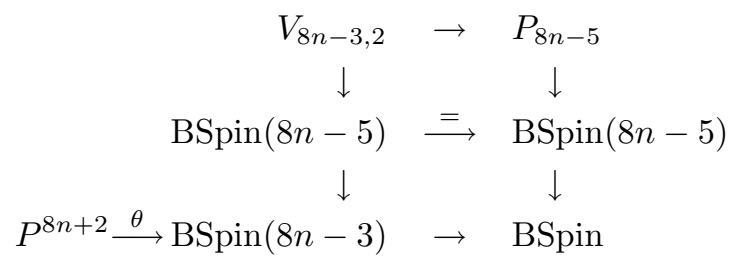


The first fiber, $V_{8 n-3,2}$, is a Stiefel manifold, and in the stable range is homotopy equivalent to the stunted real projective space $P_{8 n-5}^{8 n-4}$. The induced morphism of homotopy groups of fibers can be easily determined using the tables of [9] to be as below, where the big dots map across. It is useful to note here that these two charts fit into an exact sequence in which the third chart is $\pi_{*}\left(P_{8 n-3}\right)$.

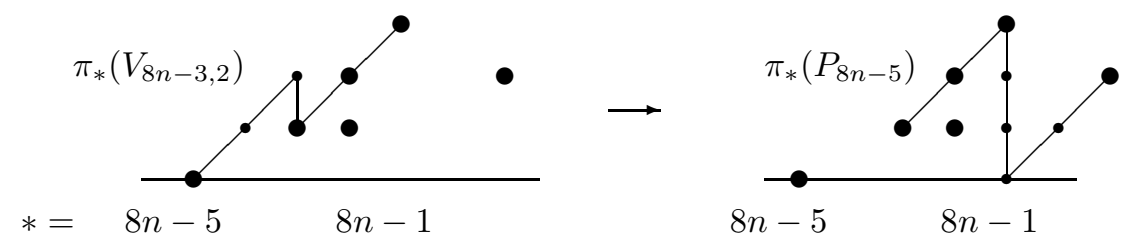

FiguRE 3.5.

The MPT for the fibration BSpin $(8 n-5) \rightarrow \operatorname{BSpin}(8 n-3)$ corresponds to the above chart of $\pi_{*}\left(V_{8 n-3,2}\right)$. We denote its spaces by $A_{i}$.

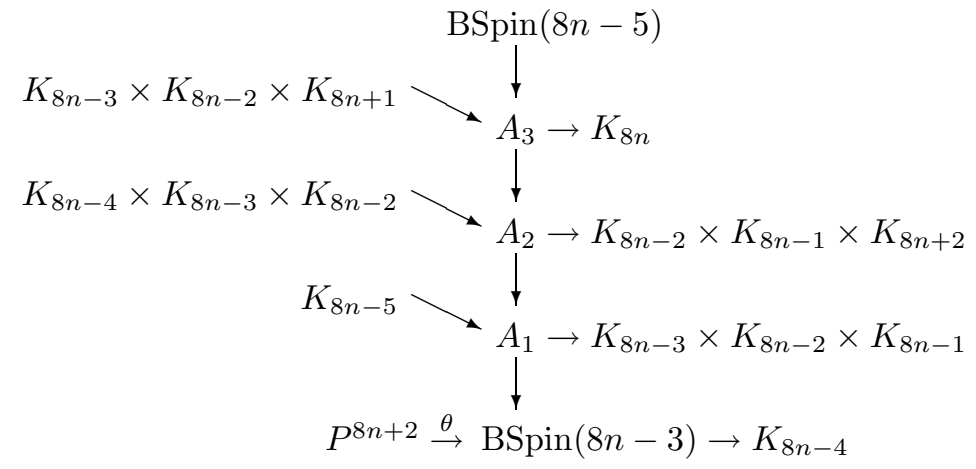

Figure 3.6 .

We need to know the relations that give rise to these $k$-invariants. We list them without listing the $w_{4}$ and $w_{8}$ which appear in some of these relations, because $w_{4}=0$ and $w_{8}=0$ for the bundle being considered here.

\begin{tabular}{|l|}
\hline$w_{8 n-4}$ \\
\hline$k_{8 n-3}^{1}: \mathrm{Sq}^{2} w_{8 n-4}$ \\
$k_{8 n-2}^{1}: \mathrm{Sq}^{2} \mathrm{Sq}^{1} w_{8 n-4}$ \\
$k_{8 n-1}^{1}: \mathrm{Sq}^{4} w_{8 n-4}$ \\
\hline$k_{8 n-2}^{2}: \mathrm{Sq}^{1} k_{8 n-2}^{1}+\mathrm{Sq}^{2} k_{8 n-3}^{1}$ \\
$k_{8 n-1}^{2}: \mathrm{Sq}^{2} k_{8 n-2}^{1}$ \\
$k_{8 n+2}^{2}:\left(\mathrm{Sq}^{4}+\mathrm{Sq}^{3} \mathrm{Sq}^{1}\right) k_{8 n-1}^{1}+\mathrm{Sq}^{6} k_{8 n-3}^{1}$ \\
\hline$k_{8 n}^{3}: \mathrm{Sq}^{3} k_{8 n-2}^{2}+\mathrm{Sq}^{2} k_{8 n-1}^{2}$ \\
\hline
\end{tabular}


Primary indeterminacy allows the following classes to be changed (if liftings to the indicated level exist):

- $f_{1}^{*}\left(k_{8 n-3}^{1}\right)$ (through $\left.K_{8 n-5}\right)$;

- $f_{2}^{*}\left(k_{8 n-2}^{2}\right)$ (through $\left.K_{8 n-3}\right)$;

- $f_{2}^{*}\left(k_{8 n+2}^{2}\right)$ (through $\left.K_{8 n+2}\right)$;

- $f_{3}^{*}\left(k_{8 n}^{3}\right)$ (through $\left.K_{8 n}\right)$.

We can also use relations in the MPT to deduce that (again, if the liftings to the indicated level exist) $f_{1}^{*}\left(k_{8 n-2}^{1}\right)=0$ and $f_{2}^{*}\left(k_{8 n-1}^{2}\right)=0$. To see the first of these, we use the relation that gives rise to $k_{8 n-1}^{2}$. This says that $\mathrm{Sq}^{2} k_{8 n-2}^{1}=0 \in H^{*} A_{1}$. Thus $\mathrm{Sq}^{2}\left(f_{1}^{*}\left(k_{8 n-2}^{1}\right)\right)=0 \in H^{*}\left(P^{8 n+2}\right)$, and so we cannot have $f_{1}^{*}\left(k_{8 n-2}^{1}\right)=x^{8 n-2}$. The equation $f_{2}^{*}\left(k_{8 n-1}^{2}\right)=0 \in H^{*} A_{2}$ follows similarly from the relation for $k_{8 n}^{3}$.

Because $w_{8 n-4}(\theta)=w_{8 n-4}(16 n \xi)=\left(\begin{array}{c}16 n \\ 8 n-4\end{array}\right) x^{8 n-4}=0 \in H^{*}\left(P^{8 n+2}\right)$, the map $\theta$ lifts to $f_{1}: P^{8 n+2} \rightarrow A_{1}$. Once we show that $f_{1}^{*}\left(k_{8 n-1}^{1}\right)=0$, we will be able to deduce our desired lifting to BSpin $(8 n-5)$, for all other $k$-invariants are either in the indeterminacy or map to 0 by relations, as described in the preceding paragraph.

The map of fibrations in (3.4) induces a map of MPTs. The element in filtration 1 in $\pi_{8 n-2}$ (fibers) maps across, as indicated in Figure 3.5. This implies that the corresponding level-1 $k$-invariant maps across; i.e., if $g_{1}: A_{1} \rightarrow E_{1}$ is the induced map of spaces in the MPTs, then $g_{1}^{*}\left(\tilde{k}_{8 n-1}^{1}\right)=k_{8 n-1}^{1}$, where we use the tilde to indicate the $k$-invariants in the MPT for $\operatorname{BSpin}(8 n-5) \rightarrow \mathrm{BSpin}$. In the paragraph after the proof of Lemma 3.3. we have observed that the map $\theta$ into BSpin lifts to BSpin $(8 n-5)$. This implies that the composite $P^{8 n-2} \stackrel{f_{1}}{\longrightarrow} A_{1} \stackrel{g_{1}}{\longrightarrow} E_{1}$ differs from a map $h_{1}$ that lifts to $\operatorname{BSpin}(8 n-5)$ by a map $\delta_{1}$ which factors through the fiber $K_{8 n-5} \times K_{8 n-1}$ of $E_{1} \rightarrow$ BSpin. The relation for $\tilde{k}_{8 n-1}^{1}$ is $\left(\mathrm{Sq}^{4}+w_{4}\right) w_{8 n-4}$. Since $\mathrm{Sq}^{4}\left(x^{8 n-5}\right)=0$ and $w_{4}(\theta)=0$, any such map $\delta_{1}$ must send $\tilde{k}_{8 n-1}^{1}$ to 0 . Since $h_{1}$ lifts, it sends all $k$-invariants to 0 . Thus

$$
0=h_{1}^{*}\left(\tilde{k}_{8 n-1}^{1}\right)+\delta_{1}^{*}\left(\tilde{k}_{8 n-1}^{1}\right)=f_{1}^{*} g_{1}^{*}\left(\tilde{k}_{8 n-1}^{1}\right)=f_{1}^{*}\left(k_{8 n-1}^{1}\right)
$$

as desired.

\section{REFERENCES}

[1] L. Astey and D. M. Davis, Nonimmersions of real projective spaces implied by BP, Bol Soc Mat Mex 24 (1979) 49-55.

[2] D. M. Davis, Some new immersions and nonimmersions of real projective spaces, AMS Contemporary Math 19 (1983) 51-64. MR 84k:57016

[3] _ Table of immersions and embeddings of real projective spaces, http://www.lehigh.edu/ dmd1/immtable

[4] - Computing $v_{1}$-periodic homotopy groups of spheres and certain compact Lie groups, Handbook of Algebraic Topology, Elsevier (1995) 993-1049. MR 97g:55017

[5] D. M. Davis and M. Mahowald, The geometric dimension of some vector bundles over projective spaces, Trans Amer Math Soc 205 (1975) 295-315. MR 51:9058

[6] S. Gitler and M. E. Mahowald, The geometric dimension of real stable vector bundles, Bol Soc Mat Mex 11 (1966) 85-107. MR 37:6922

[7] A. Haefliger, Plongements différentiable dans le domaine stable, Comm Math Helv 37 (1962) 155-176. MR 28:625

[8] M. E. Mahowald, On obstruction theory in orientable fibre bundles, Trans Amer Math Soc 110 (1964) 315-349. MR 28:620 
[9] The metastable homotopy of $S^{n}$, Mem Amer Math Soc 72 (1967). MR 38:5216

[10] B. J. Sanderson, A nonimmersion theorem for real projective space, Topology 2 (1963) 209211. MR 27:1968

[11] E. Thomas, Embedding manifolds in Euclidean space, Osaka Jour Math 13 (1976) 163-186. MR 57:13978

[12] V. Zelov, Embeddings and immersions of real projective spaces, Ph.D. thesis, Lehigh University (1997).

Department of Mathematics, Lehigh University, Bethlehem, Pennsylvania 18015

E-mail address: dmd1@lehigh.edu

Microsoft Corporation, Charlotte, North Carolina

E-mail address: vitalyze@microsoft.com 